\title{
ZUZANA MaLinovská
}

Université de Prešov

\section{La honte comme mobile narratif dans}

Pas pleurer de Lydie Salvayre ${ }^{1}$

e point de départ de ma réflexion est le sentiment que l'univers romanesque de l'auteure française contemporaine Lydie Salvayre est imprégné de honte. La honte est le trait distinctif de la plupart de ses personnages, surtout dans les premiers textes comme $L a$ Déclaration (1990), La Vie commune (1991), La Puissance des mouches (1995), La Compagnie des spectres (1997), La Conférence de Cintegabelle (1999), etc. Ce mélange d'émotions et de sentiments pénibles est souvent inscrit dans les corps des personnages. "Je ne contiens plus que la honte, ma gorge se serre, je ne sais plus parler ${ }^{2}$, se confie dès l'incipit le narrateur-protagoniste de $L a$ Déclaration, premier roman de l'auteure, qui relate l'histoire d'un homme qui sombre dans la folie après le départ de sa femme. "J'ai balbutié, j'ai rougi, j'ai eu la honte ${ }^{3}$, ajoute-t-il, tout en se définissant comme "l'enfant malade de la honte ${ }^{4}$. Mis à part les fréquentes manifestations corporelles de la honte, les textes de Lydie Salvayre frappent par la manière dont l'écrivaine introduit ses personnages dans l'univers romanesque. II n'est pas rare qu'elle les donne à voir comme des corps peu désirables, malades, suscitant la répulsion et la honte. Très

1 L'article s'inscrit dans le cadre du projet VEGA 2/0063/2016 Hyperlexikón literárnovedných pojmov a kategórií II.

${ }^{2}$ L. Salvayre, La Déclaration, Paris, Seuil, 1997, p. 10.

${ }^{3}$ Ibidem, p. 10.

${ }^{4}$ Ibidem. 
souvent l'auteure ne met en relief qu'une partie du corps, de préférence invisible et liée au fonctionnement de l'organisme. Ainsi la femme du protagoniste-narrateur de La Déclaration se résume à ses " intestins ", ses " organes détraqués ", son " estomac " ${ }^{5}$, etc. Même les parties publiques, visibles, du corps du personnage font l'objet d'une représentation dévalorisante : l'épouse a " de grosses cuisses dont la graisse tremblote $\|^{6}$, " une bouche archaïque ${ }^{7}$ qui n'est autre chose que "le début du tube digestif ${ }^{8}$. Cette vision de l'épouse n'est peutêtre pas surprenante, étant donné que la narration est focalisée sur le mari abandonné, pétri de honte et de haine. Toutefois, le narrateur voit sa mère de la même manière, présente par ses " jambes variqueuses et le ventre mou $"{ }^{9}$, "la masse visqueuse et flasque de ses organes génitaux », " la descente d'organes $»^{10}$, etc.

Les exemples ci-dessus visent à démontrer qu'au lieu de signaler les personnages par les parties publiques, nobles du corps, le point d'honneur de tout individu, Lydie Salvayre met l'accent sur les parties intimes et invisibles, " honteuses " du corps humain, comme les organes génitaux et défécatoires, les composantes du tube digestif, etc. Comme si la simple exposition de parties privées et cachées ne la satisfaisait pas, Lydie Salvayre complète cet étalage d'intimité de menus détails et précisions relatives au fonctionnement et au dysfonctionnement des parties du corps exhibées. Ainsi, la mère du protagoniste de $L a$ Déclaration souffre de "ballonnement qui plaque la vessie

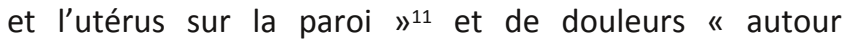
de l'anus ${ }^{12}$ qui l'obligent à consulter un proctologue.

\footnotetext{
5 Ibidem, p. 7, 10.

${ }^{6}$ Ibidem, p. 9.

7 Ibidem, p. 7.

8 Ibidem, p. 95.

${ }^{9}$ Ibidem, p. 9.

10 Ibidem, p. 35.

11 Ibidem, p. 11.

12 Ibidem, p. 35.
} 
La " mauvaise haleine ", " l'odeur [...] qui monte de [son] estomac ${ }^{13}$ caractérise sa femme, etc. Le même traitement est appliqué à bien d'autres personnages, protagonistes ou secondaires. Ainsi, Lucienne de La Conférence de Cintegabelle, "grosse pouffe ${ }^{14}$ taciturne, se résume au " broiement masticatoire et [...] faibles éructations ${ }^{15}$. Le narrateur de La Méthode Mila (2005), en train de rédiger sa thèse, est systématiquement dérangé par "les demandes urino-défécatoires ${ }^{16}$ de sa mère, et ainsi de suite, inutile de multiplier les exemples. L'acharnement de l'auteure à ne montrer que l'aspect " honteux " du corps - traduction probable de sa vision de la condition humaine inséparable de la honte - est particulièrement flagrant dans les évocations dégradantes des parties nobles. Ainsi la bouche, l'organe de la parole, le lieu privilégié par lequel on séduit et on s'affirme dans la société, devient dans La Déclaration " I'orifice buccal $\aleph^{17}$, " le début du tube digestif [...] un sphincter étroit ${ }^{18}$, etc. On peut donc constater que la représentation de l'homme réduit à son corps qui lui fait honte et qui inspire la honte constitue un des traits typiques de l'œuvre de Lydie Salvayre.

Mais la honte n'est pas uniquement une affaire de corps et de sa dépréciation; elle est souvent liée à la naissance, aux origines, au milieu familial, au lieu social. De nombreux personnages de Lydie Salvayre ont honte de leurs parents, pères autoritaires et violents aux " mains énormes renforcées par des cals ${ }^{19}$ et mères mortesvivantes subissant en silence le mauvais traitement infligé par leurs époux. Ainsi, le protagoniste-narrateur de $L a$

\footnotetext{
13 Ibidem, p. 7.

${ }^{14}$ L. Salvayre, La Conférence de Cintegabelle, Paris, Seuil, 1999, p. 35.

15 Ibidem, p. 40.

${ }^{16}$ L. Salvayre, La Méthode Mila, Paris, Seuil, 2005, p. 86.

${ }^{17}$ L. Salvayre, La Déclaration, op. cit., p. 18.

18 Ibidem, p. 95.

${ }^{19}$ L. Salvayre, La Puissances des mouches, Paris, Seuil, 1996, p. 30.
} 
Déclaration fait l'aveu de " cette honte affreuse qu'[il a] de ses parents ${ }^{20}$ : il a honte de sa mère et de son père aux " mains rouges et énormes " et au " cerveau écrasé par le vacarme de la bétonnière ${ }^{21}$, " honte de la misère de son corps, de la misère de ses nerfs, de la misère de sa vie ${ }^{22}$. Et en même temps, il a honte d'en avoir honte ${ }^{23}$. II en est de même pour Pascal, de La Puissance des mouches, honteux car trop lâche et faible pour défendre sa mère et sa sœur contre son père, macho brutal et coléreux, pour le narrateur-personnage de La Méthode Mila (2005), peu compatissant avec sa vieille mère, pour le conférencier de La Conférence de Cintegabelle constatant la mort " honteuse " de la conversation et pleurant sa femme dont il avait pourtant honte et pour bien d'autres personnages qui évoluent tous dans " un monde trouble [...] où le plaisir » cède à " la honte et au remords $»^{24}$.

On peut donc avancer que les romans et récits de Lydie Salvayre donnent à voir de multiples formes de honte intime et sociale, mais aussi historique et politique. La honte récurrente et envahissante devient invariant thématique sous-jacent, leitmotif, parfois principe de construction, voire mobile narratif de ses récits. C'est la honte qui pousse la narratrice de La Compagnie des spectres à parler à l'huissier venu faire la saisie. À la honte liée à une situation matérielle précaire s'ajoute la honte de la mère, marquée à jamais par le souvenir d'un crime honteux, l'assassinat de son jeune frère en janvier 1943 par deux zélés serviteurs du pouvoir. Dans La Puissance des mouches, le patricide peut s'expliquer par une honte viscérale jamais jugulée; cette honte conduit Pascal à raconter tour à tour au gardien de prison, au juge, au psychiatre, à l'infirmier sa vie noyée dans la honte.

\footnotetext{
20 Ibidem, p. 10.

21 Ibidem.

22 Ibidem.

23 Ibidem : " Redevenu l'enfant malade de la honte, j'ai balbutié, j'ai rougi, j'ai eu honte de ma honte ".

${ }^{24}$ L. Salvayre, La Puissances des mouches, op. cit., p. 40.
} 
Comment expliquer cette obsession de l'écrivaine pour la honte?

La honte omniprésente dans l'œuvre de Lydie Salvayre prend source dans le vécu. Dans un entretien avec Michel Houellebecq publié dans la revue Perpendiculaire, Lydie Salvayre avoue la honte ${ }^{25}$ qui pollua longtemps son enfance. La future romancière grandit dans la honte de ses origines modestes, doublée de l'inquiétude honteuse de ne jamais accéder à la maîtrise de la langue française, porte d'entrée à la réussite. Car à la maison on parle espagnol. Pour cette fille de réfugiés espagnols n'ayant jamais appris la langue de leur pays d'accueil, apprendre le français devient un défi. Défi relevé : Lydie Salvayre réussit non seulement à apprivoiser la langue française mais aussi à en faire un lieu et un moyen d'affranchissement. Et de surcroît un instrument de travail : de l'écrivain, en premier lieu, avec une bonne vingtaine de titres à son compte, et du médecin spécialisé en pédopsychiatrie. Lydie Salvayre transforme donc la honte contractée à son enfance en force motrice débouchant sur une double victoire : une ascension sociale assurée par des études de médecine (où l'appartenance à une classe défavorisée constitue un handicap) et un renom d'écrivain dont l'écriture est nourrie de honte, ce "poison de l'âme ${ }^{26}$. Pour démontrer mon point de vue, je vous propose une lecture du dernier roman de Lydie Salvayre intitulé Pas pleurer (2014), inspirée entre autres des idées de Boris Cyrulnik, notamment son Mourir de dire. La honte 27.

Prix Goncourt 2014, Pas pleurer ${ }^{28}$ est une biographie romancée, revendiquée directement par l'une des voix narratives : «Dans le récit que j'entreprends, je ne veux

${ }^{25}$ Cf. Cité d'après T. Guichard, "Lydie Salvayre, le francais pour mémoire ", [dans :] Le Matricule des Anges, 15 mai-15 juillet 1999, n²6, p. 17.

${ }^{26}$ B. Cyrulnik, Mourir de dire. La honte, Paris, Odile Jacob, 2010, p. 24.

27 Ibidem.

${ }^{28}$ L. Salvayre, Pas pleurer, Paris, Seuil, 2014. 
introduire, pour l'instant, aucun personnage inventé ${ }^{29}$. Elle est constituée de trois récits enchevêtrés dont les composantes essentielles - l'univers spatio-temporel, les personnages, les actions et les narrateurs - sont, à des degrés différents, marquées par la honte. Le récit-cadre est contemporain : situé dans un village du Sud-Ouest français, il a pour protagoniste Montse chargée également de la narration. Nonagénaire souffrant de graves troubles de la mémoire et s'exprimant dans un drôle de français mâtiné d'espagnol, Montse évoque en février 2011 l'été libertaire espagnol de 1936, " l'unique aventure de son existence ${ }^{30}$. Les événements de cet été constituent la trame du second récit. Situé dans la campagne catalane des années trente, ce récit enchâssé évoque le quotidien de la jeune Montse et de ses proches lié étroitement aux événements historiques qui ont abouti à une dictature sanguinaire de plus de trente ans. La vieille Montse adresse son discours à sa fille Lidia, double fictionnel à camouflé de l'auteur. Seconde narratrice intradiégétique, Lidia complète et rectifie I'histoire de sa mère, réfugiée espagnole installée en France depuis février 1939, tout en corrigeant son français approximatif. Le récit-cadre, un tête-à-tête intime de mère et de fille, se déroule dans l'espace sécurisant de la maison d'où la honte semble être évacuée : Montse parle sans aucune retenue et ne se préoccupe ni du bon usage du français ni des conventions de la bienséance. La vieillesse et la maladie l'aidant, elle semble débarrassée de la honte, sorte de seconde peau qui collait à sa vie et à celle de ses proches.

Enfin, le troisième récit de l'ensemble romanesque, un va-et-vient entre l'Espagne de la guerre civile et la France contemporaine, est constitué de commentaires par la fille de Montse de citations tirées de Grands cimetières sous la lune de Georges Bernanos. Ce texte très sombre

29 Ibidem, p. 15.

30 Ibidem, p. 16. 
qui est un témoignage poignant sur l'horreur de la guerre civile, rédigé par l'auteur catholique, témoin oculaire à Palma de Majorque de la violence aveugle des franquistes, assure dans Pas pleurer la fonction testimoniale et idéologique. Toutefois, complété par les remarques de Lidia alias Lydie Salvayre (née en 1948, l'année de la mort de Bernanos), il annonce également un programme artistique : la création littéraire vue comme un incessant dialogue avec les prédécesseurs. J'avance cette hypothèse vu l'intertextualité frappante que j'ai observée ${ }^{31}$ dans les textes de Lydie Salvayre. L'échange de deux auteurs que pourtant tout sépare et qui à soixante-seize ans d'intervalle évoquent les mêmes événements historiques honteux - montre que Georges Bernanos et Lydie Salvayre partagent la même vision de l'Espagne de la guerre civile : c'est un univers honteux de barbarie et d'humiliation. Et peu importe l'idéologie... Toutes les voix narratives l'affirment en montrant que des deux côtés - " d'un côté un front dit populaire composé des différentes gauches [...] de l'autre un front dit national formé de droites coalisées ${ }^{32}$ - des actes barbares étaient commis sans que leurs auteurs éprouvent le moindre sentiment de honte.

Comment expliquer cette absence de honte aboutissant à une barbarie honteuse qui envahit le pays entier ? Probablement par le contexte politique et social de l'époque. Toute situation de guerre, explique Boris Cyrulnik, fabrique des "pervertis ${ }^{33}$. Ces individus sans structure perverse de personnalité, une fois soumis aveuglément à une idéologie, perdent toute capacité d'empathie et par la suite tout sentiment de honte. Leur unique objectif est de faire à tout prix triompher leurs idées. Lydie Salvayre semble souscrire à cette vision : ses

31 Z. Malinovská, Puissances du romanesque. Regard extérieur sur quelques romans contemporains d'expression française, ClermontFerrand, Presses Universitaires Blaise Pascal, 2010, p. 88-95 et 133-140.

${ }^{32}$ L. Salvayre, Pas pleurer, op. cit., p. 106.

${ }^{33}$ B. Cyrulnik, Mourir de dire. La honte, op. cit., p. 243-247. 
personnages inspirés de personnes réelles, paysans pauvres, paisibles, respectueux des traditions immuables, peuvent, dans le contexte de la guerre, devenir " pervertis ". Esclaves d'une idéologie qui se superpose à une culture de l'honneur particulièrement forte en Espagne, culture qui condamne toute faiblesse et toute défaite même illusoires, ces personnages commettant des actes honteux ne ressentent ni remords, ni culpabilité, ni honte. Car pour eux, tout simplement, l'autre n'existe pas. Or, la honte requiert toujours la présence de l'autre : " II n'y a pas de honte quand il n'y a pas de regard de l'autre $»^{34}$.

Pour mettre en relief l'importance du regard de l'autre dans l'éveil du sentiment de honte, Lydie Salvayre opte pour un incipitex abrupto. Pas pleurer s'ouvre sur la rencontre de la protagoniste avec celui qui, contre toute attente, deviendra son beau-père. La scène montre Montse, âgée de quinze ans - c'est-à-dire un âge de grandes aspirations où le regard de l'autre est quasi vital et le sentiment de la honte culmine - qui se présente chez don Burgos, riche propriétaire terrien, pour proposer ses services comme bonne. "Elle a l'air bien modeste ${ }^{35}$, dit don Burgos à sa femme, ce que Montse, attribuant à l'autre un regard méprisant, ressent comme une grande humiliation. Issue d'une famille pauvre et confrontée au riche notable local, Montse se sent minable même si don Burgos ne la voit pas ainsi. Sa remarque anodine génère chez elle un trauma et prend la signification d'un événement extraordinaire qui jalonnera son histoire. J'appuie cette possibilité interprétative sur l'emplacement de la scène à l'incipit - l'endroit important de la prise de contact entre le lecteur et le monde représenté - et sur la répétition, tel un refrain dans le récit, de cette petite phrase. Le jour où Montse fait part de ce sentiment non

34 Ibidem, p. 247.

${ }^{35}$ L. Salvayre, Pas pleurer, op. cit., p. 13. 
partageable qu'est la honte à celui qui l'a, à son insu, provoqué, elle a toutes les chances de prendre le chemin de la résilience. Mais la résilience requiert « le tissage d'un lien affectif stable ${ }^{36}$ et Montse ne se conforme pas à cette condition : ainsi elle continue à vivre avec sa honte.

De nombreuses scènes de Pas pleurer soulignent ce côté non racontable de la honte qui isole le honteux tout en le rapprochant, paradoxalement, de l'autre. Ainsi Montse, tombée enceinte après sa première expérience sexuelle au moment où elle pensait avoir trouvé le bonheur, est à la fois seule avec sa honte et jamais seule. De retour au village natal, elle est livrée au regard écrasant et réprobateur des villageois. Car, au début du siècle dernier dans l'Espagne catholique, dans un milieu paysan de surcroît, la culture de l'honneur est particulièrement pesante. Elle impose aux filles de garder leur virginité juqu'au mariage et ordonne aux paters familias, machos autoritaires, d'y veiller. La faute de Montse qui a manqué à ce culte de l'hymen ne peut être réparée que par le mariage. L'union avec Diego, fils adoptif de don Burgos et ennemi juré de Josep, la réhabilite socialement, mais ne soulage pas sa honte inavouable doublée de la honte de ne pas aimer son mari. Ce mariage de raison n'est donc qu' « un bénéfice secondaire d'une souffrance cachée ${ }^{37}$ dont l'origine est la déchirure entre ce que Montse aspirait à être (à savoir une fille libérée, indépendante, mariée au bel inconnu qui l'a mise enceinte) et ce qu'elle est réellement devenue (abandonnée par « celui que ma sœur et moi appelons, depuis l'enfance, André Malraux ${ }^{38}$ et mariée sans amour au premier venu).

Je peux donc avancer que Lydie Salvayre connaît bien les mécanismes de la honte qu'elle arrive à mettre

${ }^{36}$ B. Cyrulnik, Mourir de dire. La honte, op. cit., p. 113.

37 Ibidem, p. 38.

${ }^{38}$ L. Salvayre, Pas pleurer, op. cit., p. 162. 
en fiction ${ }^{39}$ de manière convaincante. Dans Pas pleurer la honte sert de matière première utilisée dans la construction des actions, des personnages et de l'univers noyé dans la honte. Ainsi le personnage de Diego - que pourtant rien ne prédestine à la honte, à première vue - est pétri de honte. II se sent minable sous le regard de l'autre, que ce soit Montse, son frère Josep, voire la communauté des paysans. Enfant illégitime, adopté par un riche couple, Diego pourtant entouré d'affection a honte de ses origines, des rumeurs sur sa mère biologique, de ses cheveux roux, de son statut de " seigneur ". II cache la perception dégradée de soi sous un masque de dureté et de cynisme, particulièrement flagrant dans ses relations avec ses parents, surtout sa mère adoptive Doña Sol. En créant ce personnage féminin, c'est comme si Lydie Salvayre soulignait encore davantage les déterminants socioculturels de la honte : autre variante de femme écrasée par la honte, Doña Sol ne correspond pas aux canons culturels imposant aux femmes mariées d'enfanter. En revanche, la honte des parents de Montse - ce père autoritaire, peu enclin aux " palabres affectueuses [...] domaine exclusif des femmes ${ }^{40}$ et cette mère soumise - tous les deux incarnation par excellence de la culture de l'honneur en vigueur, vient d'un fort sentiment d'infériorité. Les rares scènes réunissant les parents de Montse et de Diego dévoilent deux mondes différents qui s'observent et se jugent dans le mutisme. À ce sujet, la scène du repas de noces où la jeune Montse " paralysée de honte " regardant son père boire et devenir obscène, découvre sous le masque de "l'ogre ", du "tyran ", du " plus redoutable des hommes " un pauvre type est éloquente ${ }^{41}$. Sa honte d'avoir transgressé le code culturel imposé aux filles se double ici de la honte de ses origines et en même temps de la honte d'en avoir honte.

\footnotetext{
${ }^{39}$ Dans notre cas de figure, la fiction est fortement inspirée du vécu.

${ }^{40}$ L. Salvayre, Pas pleurer, op. cit., p. 222.

${ }^{41}$ Ibidem, p. 190-193.
} 
Sont scupltés de honte également les autres personnages de Pas pleurer, excepté peut- être don Burgos, sorte de sage modéré. À titre d'exemple, je peux citer doña Pura, avec la honte inscrite dans un corps sec de vieille fille rigide et pieuse, car sa pensée, en contradiction avec les injonctions bibliques et la pureté inscrite dans son nom, dévoile la cruauté éhontée de la dévote pudibonde : incapable d'aimer son prochain, elle lui souhaite la mort. Elle déteste notamment les "rouges " qui " offensent mortellement son bon Dieu $»^{42}$. Le frère de Montse, Josep, est le seul qui crie la honte de sa condition de dominé et la transforme en colère exprimée par ses actes.

Trois personnages de Pas pleurer ont toutefois un statut différent dans l'univers fictionnel : Montse, Lidia, alter ego de l'auteur, et Georges Bernanos, car ils rompent le silence honteux et deviennent narrateurs pour faire part de leur honte. Lidia-Lydie le fait par le biais de l'histoire de sa mère, Georges Bernanos - " blessé " par "le scandale d'une Église qui tapine avec les militaires $»^{43}$ - sous forme de témoignage direct. Ce fervent catholique, ancien militant de l'Action française sympathisant avec le mouvement franquiste, horrifié par le fanatisme et révolté par la complicité de l'Église qui justifie les massacres des innocents rédige son célèbre essai ${ }^{44}$ pour crier sa honte devenue rage. Et pour avouer son erreur...

Inhérente à l'univers et aux personnages de Pas pleurer, la honte est le déclencheur des actions romanesques formant la petite histoire de Montse inséparable de la grande Histoire. Moteur des actions, la honte est le véritable ciment liant les personnages entre eux, en les liant en même temps à un univers spatio-temporel baignant dans la honte. Mais la honte est un mobile

\footnotetext{
42 Ibidem, p. 20.

43 Ibidem, p. 91.

44 Publié dans la revue des dominicains, Les Grands cimetières sous la lune, provoque la mise à prix par le général Franco de la tête de G. Bernanos, obligé de s'exiler.
} 
narratif avant toute chose : c'est l'impossibilité de continuer à taire sa honte longtemps vécue comme inavouable qui pousse la vieille Montse à raconter son histoire, c'est la honte également qui conduit Bernanos à dénoncer la barbarie qui lui fait honte. Enfin, c'est la honte en quête de la résilience qui dicte à Lidia-Lydie de prendre la parole. En chargeant les trois personnages de la narration, c'est comme si Lydie Salvayre nous montrait l'issue : I'unique moyen susceptible d'atténuer la honte est de ne pas la taire.

En vue de mettre en relief la puisssance réparatrice de la parole, l'auteur opte pour une forme de "roman parlé " où s'entremêlent plusieurs voix: celle de Montse, truculente, captivante, parfois hilarante, malgré la gravité du sujet abordé, celle de Bernanos, grave, accusatrice et plaidante, celle de Lidia, acquiesçante, contestante, rectifiante. Le style intentionnellement bâclé, avec des ruptures dans la construction des phrases, des répétitions, c'est-à-dire une manière de faire que l'on pourrait appeler honteuse, souligne encore davantage la thématique de la honte. Celle-ci est mise en évidence également par une langue qui pourrait être qualifiée de langue de la honte, car libérée des contraintes, relâchée, parfois vulgaire ${ }^{45} \ldots$

Lydie Salvayre connaît bien la complexité de l'être humain qu'elle représente, s'inspirant de Blaise Pascal, dans ses contradictions, petit et grand, éhonté et honteux. C'est également dans ses contradictions qu'elle voit la honte dont elle dévoile les multiples causes externes (sociales et culturelles, comme la misère, la guerre, les préjugés, les conventions, etc.) et internes (les prédispositions génétiques, comme par exemple chez Diego, etc.). Substance toxique, frein à l'épanouissement personnel, important générateur de mal de vivre à la

45 D'après Lydie Salvayre, l'usage du juron en Espagne est, contrairement à son usage en France, courant et prolifique ( $C f$. M. Grosjean, " Lydie Salvayre injecte de la joie libertaire dans I'horreur franquiste ", [dans :] Tribune de Genève, 4 novembre 2014). 
puissance dévastatrice, voire déclencheur de pathologies aux conséquences désastreuses, la honte vaincue peut cependant relancer une nouvelle existence.

Mise en garde contre les abus et débordements, Pas pleurer de Lydie Salvayre est un texte universel et hautement actuel, révélateur de notre époque. 


\section{bibliographie}

Bernanos G., Les Grands cimetières sous la lune, Paris, Plon, 1938.

Cyrulnik B., Mourir de dire. La honte, Paris, Odile Jacob, 2010.

Grosjean M., "Lydie Salvayre injecte de la joie libertaire dans l'horreur franquiste ", [dans :] Tribune de Genève, 4 novembre 2014.

Guichard T., "Lydie Salvayre, le français pour mémoire ", [dans :] Le Matricule des Anges, 15 mai-15 juillet 1999, n 26.

Lasserre A., "Le roman français contemporain aux prises avec l'Histoire : Dora Bruder de Patrick Modiano et La Compagnie des spectres de Lydie Salvayre ", [dans :] Sites, 2002, vol. 6, n².

Louichon B., Lydie Salvayre : parler au nom d'Olympe. Nouvelles écrivaines nouvelles voix ?, New York-Amsterdam, Rodopi, 2002.

Malinovská Z., Puissances du romanesque. Regard extérieur sur quelques romans contemporains d'expression française, Clermont-Ferrand, Presses Universitaires Blaise Pascal, 2010.

Martin J.-P., La Honte. Réflexions sur la littérature, Paris, Seuil, 2006.

Salvayre L., La Puissances des mouches, Paris, Seuil, 1996.

Salvayre L., La Déclaration, Paris, Seuil, 1997.

Salvayre L., La Conférence de Cintegabelle, Paris, Seuil, 1999.

Salvayre L., Pas pleurer, Paris, Seuil, 2014.

Salvayre L., La Méthode Mila, Paris, Seuil, 2005.

\section{abstract}

\section{Shame as the engine of the narrative in Lydie Salvayre's} Pas pleurer

The novel Pas pleurer (2014, No crying) by Lydie Salvayre, working as a psychoanalyst for a long time, can be read as a novel on shame. Textual analysis shows that shame - depicted in accordance with the theses of a psychiatrist Boris Cyrulnik - is the main topic of the narrative portrazing the effects of historical events on the lives of the individuals. The author uses shame as a means of constructing the novel, the shame being the glue that holds the structure together, determining the space and time, shaping the protagonists and their stories. Shame also functions as the starter and the engine of the narrative. The narrators-protagonists, who speaking about the "shameful events", are those who overcome their own feelings of shame by telling their stories. Timely and universal, the novel points to the therapeutic function of literature, thus leaving it open for scientists from other areas of research.

\section{keywords}

shame, narrative, engine of the narrative, Lydie Salvayre, Boris Cyrulnik 


\section{zuzana malinovská}

Professeur des universités à l'Institut d'Etudes romanes de la Faculté des Lettres de l'Université de Prešov en Slovaquie, Zuzana Malinovská enseigne l'histoire de la littérature française, la traduction littéraire et juridique. Ses recherches portent sur le roman contemporain français et francophone, la critique de traduction et la théorie littéraire. Elle a publié trois monographies et de nombreux articles parus dans des revues spécialisées en France, Slovaquie, République tchèque, Hongrie, Pologne, Turquie et au Canada. 